\title{
Stellar abundances tracing the formation of the Galactic Bulge
}

\author{
Beatriz Barbuy ${ }^{1}$, Manuela Zoccali ${ }^{2}$, Sergio Ortolani ${ }^{3}$, Vanessa Hill ${ }^{4}$, \\ Alvio Renzini ${ }^{5}$, Jorge Meléndez ${ }^{6}$, Anita Gómez ${ }^{4}$, Martin Asplund ${ }^{7}$, \\ Dante Minniti $^{2}$, Eduardo Bica ${ }^{8}$, and Alan Alves-Brito ${ }^{1}$ \\ ${ }^{1}$ Universidade de São Paulo, IAG, Rua do Matão 1226 \\ São Paulo 05508-900, Brazil \\ email: barbuy@astro.iag.usp.br \\ ${ }^{2}$ Universidad Catolica de Chile, Department of Astronomy \& Astrophysics, Casilla 306, \\ Santiago 22, Chile \\ email: mzoccali@astro.puc.cl, dante@astro.puc.cl \\ ${ }^{3}$ Università di Padova, Dipartimento di Astronomia, Vicolo dell'Osservatorio 2, I-35122 \\ Padova, Italy \\ email: sergio.ortolani@unipd.it \\ ${ }^{4}$ Observatoire de Paris-Meudon, 92195 Meudon Cedex, France \\ email: Vanessa.Hill@obspm.fr, anita.gomez@obspm.fr \\ ${ }^{5}$ Osservatorio Astronomico di Padova, Vicolo dell'Osservatorio 5, I-35122 Padova, Italy \\ email: alvio.renzini@oapd.inaf.it \\ ${ }^{6}$ Centro de Astrofísica da Universidade de Porto, Rua das Estrelas, 4150-762 Porto, Portugal \\ email: jorge@astro.up.pt \\ ${ }^{7}$ Max Planck Institute for Astrophysics, Postfach 1317, 85741 Garching, Germany \\ email: asplund@MPA-Garching.MPG.DE \\ ${ }^{8}$ Universidade Federal do Rio Grande do Sul, Departamento de Astronomia, CP 15051, Porto \\ Alegre 91501-970, Brazil e-mail: bica@if.ufrgs.br
}

\begin{abstract}
The metallicity distribution and abundance ratios of the Galactic bulge are reviewed. Issues raised by different groups in recent work, in particular the high metallicity end, a comparison between the oxygen abundances derived from different indicators, the [OI] $630 \mathrm{~nm}$ and IR $\mathrm{OH}$ lines, and the issue of measuring giants vs. dwarfs, are discussed. Finally, abundances in bulge globular clusters are briefly described.
\end{abstract}

Keywords. Galaxy: abundances, bulge, stellar content, globular clusters

\section{Introduction}

A debate concerning the nature of the Galactic bulge has been going on over a decade. Kormendy \& Kennicutt (2004), Binney (2007; 2008, this conference) consider that classical bulges are the result of mergers and can be found in Sa, Sb spirals, and that these are elliptical galaxies-like that happen to have conspicuous disks, whereas later type spirals have pseudo bulges, formed from disks that develop a bar. Still, there are two different views on this: Sellwood (this conference) for example, forms bulges from bars made of stars, whereas Combes (2007) for example, forms bars from gas. It is then expected that the bulge stellar population should be similar to the inner parts of the disk from which they formed (e.g. Athanassoula 2007).

Observational studies of stellar populations from Colour-Magnitude Diagrams (CMDs) and spectroscopic abundances indicate, so far, that the bulge is old and metal-rich, and $\alpha$-enhancement indicates early fast formation. 


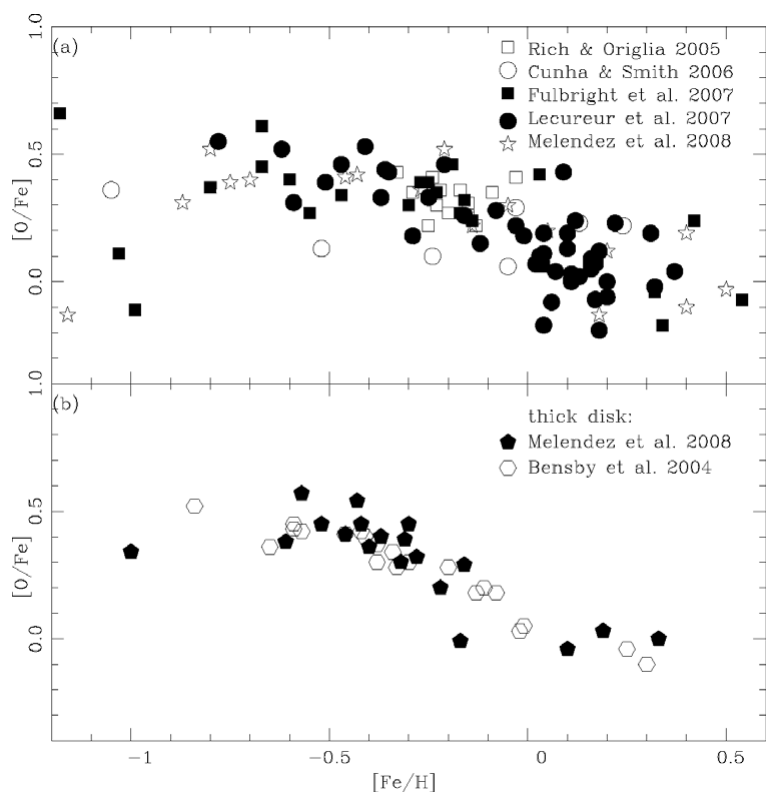

Figure 1. $[\mathrm{O} / \mathrm{Fe}]$ vs. $[\mathrm{Fe} / \mathrm{H}]$ : (a) for bulge giants analysed by Rich \& Origlia (2005), Cunha \& Smith (2006), Zoccali et al. (2006), Fulbright et al. (2007) and Meléndez et al. (2008); (b) for thick disk stars, with dwarfs from Bensby et al. (2004) and giants by Meléndez et al. (2008).

Ortolani et al. (1995), Feltzing \& Gilmore (2000), and Zoccali et al. (2003) have shown that the field bulge stars at the Baade's Window are old, since their CMDs are very similar to those of the old metal-rich globular clusters NGC 6528 and NGC 6553, which in turn show similar ages to the old metal-rich thick disk globular cluster 47 Tuc (see Alves-Brito et al. 2005).

Samples of bulge field giants studied by Rich \& Origlia (2005), Fulbright et al. (2006, 2007), Cunha \& Smith (2006), Zoccali et al. (2006, 2008), Lecureur et al. (2007), Meléndez et al. (2008) include targets from Baade's Window (BW) only, except for Zoccali et al. (2006) and Lecureur et al. (2007) who observed 3 other fields besides BW.

In short, the results so far indicate that the bulge is old, but modellers tend to believe that we are gathering data from parts not much affected by the dynamical evolution of the bar.

\section{Abundance ratios}

In recent years, abundance ratios for bulge field giants were reported by Fulbright et al. (2007), Cunha \& Smith (2006), Zoccali et al. (2006), Lecureur et al. (2007) and Meléndez et al. (2008). In particular the oxygen abundance derived by all these groups indicate an enhancement of oxygen relative to iron as shown in Fig. 1a. The oxygen enhancement is found by these 5 different groups, and these results use both the forbidden [OI] $630 \mathrm{~nm}$ line and infrared $\mathrm{OH}$ lines; therefore the oxygen enhancement appears to be a robust result. Concerning the results by Zoccali et al. (2006) and Lecureur et al. (2007) it is important to recall that $\mathrm{O}$ and $\mathrm{Mg}$ abundances were derived in four fields, and no difference was found between them.

As for the comparison with the thick disk, Zoccali et al. (2006) compared the bulge data to the thick disk dwarfs by Bensby et al. (2004), whereas Meléndez et al. (2008) compared to thick disk giants analysed in the same way as their sample bulge giants. 


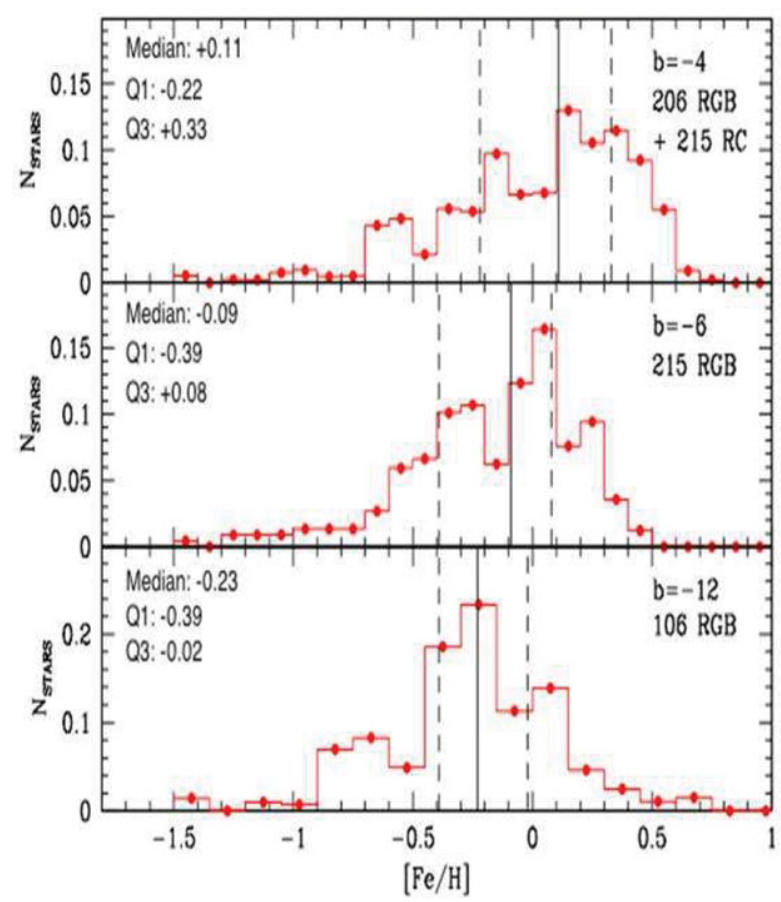

Figure 2. Metallicity distribution in 3 fields along the bulge minor axis (Zoccali et al. 2007; 2008).

Zoccali et al. (2006) found that the bulge is more oxygen-enhanced than the thick disk, whereas Meléndez et al. (2008) found that the oxygen abundances of bulge and thick disk stars overlap each other. The oxygen abundances for thick disk stars are shown in Fig. 1b; it appears that they are not very different, even if one sample concerns dwarfs and the other concerns giants. Therefore, from presently available data, the oxygen abundances of bulge stars are either slightly enhanced, or coincident with the thick disk.

$\mathrm{Mg}$ is also enhanced, and shows an overabundance relative to the thick disk (Fulbright et al. 2007; Lecureur et al. 2007). An enhancement is also found for the other $\alpha$-elements Si, Ca and Ti by Fulbright et al. (2007). However Meléndez et al. (priv. comm.) find in their sample similar abundances of $\mathrm{Mg}, \mathrm{Si}, \mathrm{Ca}$, Ti for their bulge and thick disk stars.

An issue that will have to be studied in detail as well is that some discrepancies are found between Near-Infrared and optical data, in particular this seems to be clear for Ca abundances.

Given the importance of such studies in terms of their interpretation of bulge formation, a huge effort on this would be necessary to confirm either of the conclusions.

\section{Metallicity distribution}

Zoccali et al. (2008) present the metallicity distribution for a large sample of about 800 bulge giants, observed in four fields, using FLAMES (Pasquini et al. 2000). The fields are located at: Baade's Window: $1 .{ }^{\circ} 14,-6.02, \mathrm{E}(\mathrm{B}-\mathrm{V})=0.46$; NGC 6553 field: $5.25,-3.02$, $\mathrm{E}(\mathrm{B}-\mathrm{V})=0.70$; Field at $-6^{\circ}: 0.21,-6.02, \mathrm{E}(\mathrm{B}-\mathrm{V})=0.48$; Blanco field at $-12^{\circ}: 0.00$, $-12.00, \mathrm{E}(\mathrm{B}-\mathrm{V})=0.20$.

In the BW field, two clear peaks are seen around $[\mathrm{Fe} / \mathrm{H}]=+0.3$, and -0.25 . Progressively along the minor axis, at $-6^{\circ}$ and $-12^{\circ}$, the peak at $[\mathrm{Fe} / \mathrm{H}]=+0.3$ tends to disappear as shown in Fig. 2 (Zoccali et al. 2007, 2008). We see therefore a gradient of stellar 
populations. Kinematical studies of this sample are under way, in order to better understand this most metal-rich stellar population (A. Gómez et al., in preparation).

The high end of the metallicity distribution: The Zoccali et al. (2008) metallicity distributions for the four fields give a highest metallicity at $[\mathrm{Fe} / \mathrm{H}] \approx+0.5$. The same had been found previously for the samples of 11 stars by McWilliam \& Rich (1994), and 27 giants analysed by Fulbright et al. (2006). A recalibration of Sadler et al. (1996)'s results by the latter authors indicated, however that the high end of the metallicity distribution is at $[\mathrm{Fe} / \mathrm{H}]=+1.0$.

We believe that the metallicities derived by Sadler et al. (1996), based on indices, are unreliable for the coolest and most metal-rich giants, due to the presence of $\mathrm{TiO}$ bands. $\mathrm{MgFe}, \mathrm{Mg}_{2}$ and $\mathrm{Fe}$ indices are no longer viable if $\mathrm{TiO}$ bands dominate the spectrum.

On the other hand, Johnson et al. (2007, 2008) and Cohen et al. (2008) derived metallicities and abundances for 3 dwarfs in the Galactic bulge, with spectra obtained with high $\mathrm{S} / \mathrm{N}$ favoured by a microlensing effect. The found $[\mathrm{Fe} / \mathrm{H}]=+0.5$ for the 3 dwarfs. They argue that it would be too much of a coincident to have 3 stars at the high metallicity end, so that this might be the mean metallicity of the bulge, and further argue that such stars do not become giants, which would explain the metallicity distribution by Zoccali et al. (2008). However, extreme horizontal branch stars (EHBs) should be seen in the CMDs, which is not the case. Besides, stellar evolution modellers argue that there is no such effect.

Finally, it is interesting to compare the bulge data to bulge-like dwarfs of the solar neighbourhood, which are old (around $10 \mathrm{Gyr}$ ), show pericentric distances down to $\mathrm{R}_{\text {per }}$ $\approx 3.5 \mathrm{kpc}$, and very low maximum height $\mathrm{Z}_{\max }$. We believe that these stars are outer bulge stars accelerated by the bar towards the solar neighbourhood. In Pompeia et al. (2008) it is shown that $\alpha$-element abundances are intermediate between the bulge and the thick disk.

\section{Bulge globular clusters}

We have identified a number of Blue Horizontal Branch (BHB) clusters with metallicities around $[\mathrm{Fe} / \mathrm{H}] \approx-1.0$. Within $10^{\circ} \times{ }^{\circ}$ and $\mathrm{R}<4 \mathrm{kpc}$ of the Galactic center, 36 clusters are found, half of them metal-rich $([\mathrm{Fe} / \mathrm{H}]>-0.6)$ and the other half are metal-poor. Around 8 of them show the BHB with intermediate metallicity. From the detailed analyses of 3 of them, namely HP 1 (Barbuy et al. 2006), NGC 6558 (Barbuy et al. 2007), and NGC 6522 (Barbuy et al. 2008, in preparation), the abundance patterns are very similar among them - see Fig. 3, and different from the halo pattern. The unique very metal-poor cluster in the bulge analysed so far, Terzan 4 (Origlia \& Rich 2004) shows a similar pattern to the halo.

We suggest that Terzan 4 could be a "normal halo" cluster, whereas the BHB intermediate metallicity ones might be a typical early bulge sample.

\section{Planetary Nebulae}

A comparison of the latest results on oxygen abundances in bulge giants and bulge Planetary Nebulae is presented in Chiappini et al. (2008). As previously found already, a difference of 0.2 dex with bulge giants showing a higher value, is confirmed. A detailed analysis of the uncertainties involved in the $\mathrm{O} / \mathrm{H}$ values for stars, and uncertainties in Planetary Nebulae studies, indicates that the results can be considered compatible. 


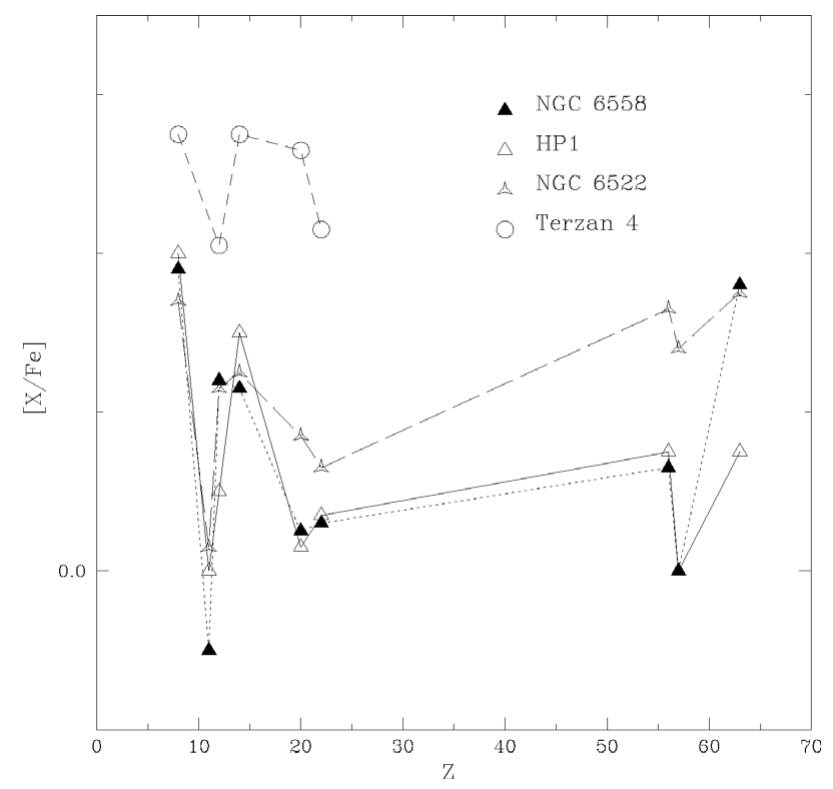

Figure 3. Abundance pattern of metal-poor bulge globular clusters: Terzan 4 with $[\mathrm{Fe} / \mathrm{H}]=-1.6$ (Origlia \& Rich 2004), and HP 1, NGC 6558, NGC 6522 with $[\mathrm{Fe} / \mathrm{H}] \approx-1.0$ (Barbuy et al. 2006, 2007, 2008).

\section{Conclusions}

Stellar populations studied so far in the bulge are old, as indicated by Colour-Magnitude Diagrams typical of $\sim 0.8 \mathrm{M}_{\odot}$ stars of ages around 12 Gyr. The $\alpha$-element Oxygen and Magnesium to iron ratios show an enhancement, indicating an early fast chemical enrichment, dominated by SNe type II.

In the very central parts of the Galaxy, within a few pc, a young population is seen, but no sign of such a young component is detected in other fields.

We recognize that more fields should be investigated, in order to have a better coverage in latitude and longitude. On the other hand, there are not that many windows, and reddening is very high towards the more central regions.

Given that we do not see signs of a younger bar population, we suggest that either have we an old bulge, and the peanut-shape bulge/bar, if younger, should be traced through abundance ratios still to be detected, or the bulge and old thick disk should have formed from a bar formed at early times.

Finally, we identify the possibly oldest globular clusters in the Galaxy, showing intermediate metallicity combined with a Blue Horizontal Branch. Should these old globulars have formed early in pre-bulge central parts, or accreted from dwarf galaxies?

\section{References}

Alves-Brito, A., Barbuy, B., Ortolani, S., Momany, Y., \& Hill, V. et al. 2005, A $\& A$ 435, 657 Athanassoula, E. 2007, in Formation and Evolution of Galaxy Bulges, IAU Symp. 245, eds. M. Bureau, E. Athanassoula, \& B. Barbuy, Cambridge University Press, p. 93

Barbuy, B., Zoccali, M., Ortolani, S., Momany, Y., \& Minniti, D. et al. 2006, A\& A 449, 349

Barbuy, B., Zoccali, M., Ortolani, S., Hill, V., \& Renzini, A., et al. 2007, AJ 134, 1613

Barbuy, B., Zoccali, M., Ortolani, S., Hill, V., \& Renzini, A., et al. 2008, A\&SA in preparation Bensby, T., Feltzing, S., \& Lundström, I. 2004, A\&A 415, 155 
Binney, J. 2007, in Formation and Evolution of Galaxy Bulges, IAU Symp. 245 eds. M. Bureau, E. Athanassoula \& B. Barbuy, p. 455

Chiappini, C., Górny, S. K., Stasińska, G., \& Barbuy, B. 2008, A\& $A$ in preparation

Cohen, J. G., Huang, W., Udalski, A., Gould, A., \& Johnson, J. 2008, arXiv 0801.3264

Combes, F. 2007, in Formation and Evolution of Galaxy Bulges, IAU Symp. 245, eds. M. Bureau, E. Athanassoula, \& B. Barbuy, Cambridge University Press, p. 149

Cunha, K. \& Smith, V. V. 2006, ApJ 651, 491

Feltzing, S. \& Gilmore, G. 2000, A\& $A, 355,949$

Fulbright, J. P., McWilliam, A., \& Rich, R. M. 2006, ApJ 636, 821

Fulbright, J. P., McWilliam, A., \& Rich, R. M. 2007, ApJ 661, 1152

Johnson, J. A., Scott Gaudi, B., Sumi, T., Bond, I. A., \& Gould, A. 2008, arXiv 0801.2159

Johnson, J. A., Gal-Yam, A., Douglas, L., Simon, J. D., Udalski, A., \& Gould, A. 2007, ApJ $655, \mathrm{~L} 33$

Kormendy, J. \& Kennicutt, R. 2004, ARA\&A 42, 603

Lecureur, A., Hill, V., Zoccali, M., Barbuy, B., Gómez, A., Minniti, D., Ortolani, S. \& Renzini, A. $2007, A \& A 465,799$

McWilliam, A. \& Rich, R. M. 1994, ApJS 91, 749

Meléndez, J., Asplund, M., Alves-Brito, A., Cunha, K., \& Barbuy, B. et al. 2008, A\&A, 484, L21

Origlia, L. \& Rich, R. M., 2004, AJ 127, 3422

Ortolani, S., Renzini, A., Gilmozzi, R., Marconi, G., Barbuy, B., Bica, E., \& Rich, R. M., 1995, Nature 377,701

Pasquini, L., Gerardo, A., Allaert, E., Ballester, P., \& Biereichel, P. et al. 2000, SPIE 4008, 129

Pompéia, L., Barbuy, B., Gustafsson, B., \& Grenon, M. 2008, ApJ in preparation

Rich, R. M. \& Origlia, L. 2005, ApJ 634, 1293

Rich, R. M., Howard, C., Reitzel, D. B., Zhao, H.-S., \& de Propis, R. 2007, in Formation and Evolution of Galaxy Bulges, IAU Symp. 245, eds. M. Bureau, E. Athanassoula, \& B. Barbuy, Cambridge University Press, p. 333

Sadler, E., Rich, R. M., \& Terndrup, D. M. 1996, AJ 112, 171

Zoccali, M., Renzini, A., Ortolani, S., Greggio, L., Saviane, I., Cassisi, S., Rejkuba, M., Barbuy, B., Rich, R. M., \& Bica, E. 2003, A\&A 399, 931

Zoccali, M., Lecureur, A., Barbuy, B., Hill, V., Renzini, A., Minniti, D., Momany, Y., Gómez, A., \& Ortolani, S. 2006, A\&\&A 457, L1

Zoccali, M., Lecureur, A., Barbuy, B., Hill, V., \& Renzini, A. et al. 2007, in Stellar Populations as Bulding Blocks of Galaxies, IAU Symp. 245, eds. A. Vazdekis \& R.F. Peletier, Cambridge University Press, p. 73

Zoccali, M., Hill, V., Lecureur, A., Barbuy, B., \& Renzini, A. et al. 2008, A\&SA 486, 177 